December 14, 2018 21:24 WSPC/INSTRUCTION FILE BU-HEPP-04-08

International Journal of Modern Physics A

(C) World Scientific Publishing Company

\title{
THRESHOLD CORRECTIONS IN PRECISION LHC PHYSICS: $\mathrm{QED} \otimes \mathrm{QCD}$
}

\author{
B.F.L. WARD \\ Department of Physics, Baylor University, One Bear Place \#97316 \\ Waco, Texas 76798-7316, USA \\ C. GLOSSER \\ Department of Physics, Southern Illinois University, Box 1654 \\ Edwardsville, IL 62026-1654, USA \\ S. JADACH \\ Institute of Nuclear Physics, ul. Radzikowskiego 152 \\ 31-342 Kraków, Poland \\ Theory Division, CERN \\ CH-1211 Geneva 23, Switzerland \\ S. A. YOST \\ Department of Physics, Baylor University, One Bear Place \#97316 \\ Waco, Texas 76798-7316, USA
}

\begin{abstract}
With an eye toward LHC processes in which theoretical precisions of $1 \%$ are desired, we introduce the theory of the simultaneous YFS resummation of QED and QCD to compute the size of the expected resummed soft radiative threshold effects in precision studies of heavy particle production at the LHC. Our results show that both QED and QCD soft threshold effects must be controlled to be on the conservative side to achieve such precision goals.
\end{abstract}

Keywords: QCD; QED; Resummation.

\section{Introduction}

At the LHC/ILC, the precision requirements for soft multiple gluon $(\mathrm{n}(\mathrm{g}))$ effects will be even more demanding than at FNAL, where the uncertainty on $m_{t}$ [1], $\delta m_{t}=4.3 \mathrm{GeV}$, receives a soft $\mathrm{n}(\mathrm{g})$ uncertainty $\sim 2-3 \mathrm{GeV}$, and soft $\mathrm{n}(\mathrm{g}) \mathrm{MC}$ exponentiation results will be an important part of the necessary theory - YFS exponentiated $\mathcal{O}\left(\alpha_{s}^{2}\right) L$ calculations, in the presence of parton showers, on an eventby-event basis.

As many authors [2] prepare the necessary results that would lead to such a precision on QCD for LHC processes, the QED corrections need to be addressed as well. Estimates by Refs. [3-7] show that one gets few per mille effects from QED 
corrections to structure function evolution. In this paper, estimate the size of QED corrections at threshold at the LHC.

Treating simultaneously QED and QCD in the respective YFS $[8,9]$ exponentiation we discuss threshold effects at the LHC in the candidate luminometry $[10,11]$ processes $p p \rightarrow V+n(\gamma)+m(g)+X \rightarrow \bar{\ell} \ell^{\prime}+n^{\prime}(\gamma)+m(g)+X$, where $V=W^{ \pm}, Z$, and $\ell=e, \mu, \ell^{\prime}=\nu_{e}, \nu_{\mu}(e, \mu)$ respectively for $V=W^{+}(Z)$, and $\ell=\nu_{e}, \nu_{\mu}, \ell^{\prime}=e, \mu$ respectively for $V=W^{-}$.

\section{YFS Theory and its Extension to QCD}

In Refs. [9] the renormalization group improved YFS theory [12] for $e^{+}\left(p_{1}\right) e^{-}\left(q_{1}\right) \rightarrow$ $\bar{f}\left(p_{2}\right) f\left(q_{2}\right)+n(\gamma)\left(k_{1}, \cdot, k_{n}\right)$ is realized by Monte Carlo methods, where the respective cross section $d \sigma_{\exp }$ and all of the attendant IR functions, $\{B, \tilde{B}, D, \tilde{S}\}$, and hard photon residuals,$\left\{\bar{\beta}_{n}\left(k_{1}, \ldots, k_{n}\right)\right\}$, are specified in Refs. [9]. In Refs. [13,14] we have extended the YFS theory to QCD: the net result is that in the analogous YFS theory we have the replacements $2 \alpha \operatorname{Re} B+2 \alpha \tilde{B} \rightarrow S U M_{I R}(Q C D), D \rightarrow D_{\mathrm{QCD}}$, and $\bar{\beta}_{n}\left(k_{1}, \ldots, k_{n}\right) \rightarrow \tilde{\bar{\beta}}_{n}\left(k_{1}, \ldots, k_{n}\right)$, where the QCD YFS functions are defined in Ref. [13] and the gluon residuals [13] $\tilde{\bar{\beta}}_{n}\left(k_{1}, \ldots, k_{n}\right)$ are free of all infrared divergences to all orders in $\alpha_{s}(Q)$. The genuine non-Abelian IR physics is encoded [13] here in the $\tilde{\bar{\beta}}_{j}$. The YFS resummation which we discuss here is fully consistent with that of Refs. [15,16]. See Ref. [17] for more discussion of this point.

\section{Extension to QED $\otimes$ QCD and QCED}

Simultaneous exponentiation of QED and QCD higher order effects gives [17]

$$
\begin{aligned}
& d \hat{\sigma}_{\exp }=e^{\mathrm{SUM}_{\mathrm{IR}}(\mathrm{QCED})} \sum_{m, n=0}^{\infty} \int \prod_{j_{1}=1}^{m} \frac{d^{3} k_{j_{1}}}{k_{j_{1}}} \prod_{j_{2}=1}^{n} \frac{d^{3} k_{j_{2}}^{\prime}}{k_{j_{2}}^{\prime}} \int \frac{d^{4} y}{(2 \pi)^{4}}
\end{aligned}
$$

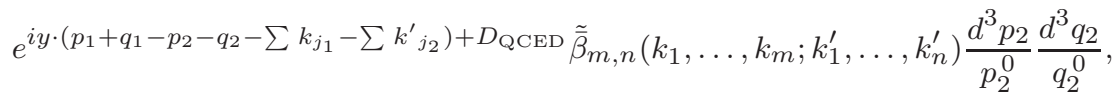

where the new YFS functions, $S U M_{I R}(Q C E D), D_{\mathrm{QCED}}$ and $\tilde{\bar{\beta}}_{m, n}\left(k_{1}, \ldots, k_{m} ; k_{1}^{\prime}, \ldots, k_{n}^{\prime}\right)$, where the latter has $m$ hard gluons and $n$ hard photons, are defined in Ref. [17]. The infrared algebra QCED [17] obtains: the average Bjorken $x$ values for the QED and QCD emissions imply [17] that QCD dominant corrections happen an order of magnitude earlier than those for QED so that the leading $\tilde{\bar{\beta}}_{0,0}^{(0,0)}$-level gives a good estimate of the size of the effects we study.

\section{QED $\otimes$ QCD Threshold Corrections at the LHC}

For the basic formula (we use the standard notation here [17])

$$
d \sigma_{\exp }\left(p p \rightarrow V+X \rightarrow \bar{\ell} \ell^{\prime}+X^{\prime}\right)=\sum_{i, j} \int d x_{i} d x_{j} F_{i}\left(x_{i}\right) F_{j}\left(x_{j}\right) d \hat{\sigma}_{\exp }\left(x_{i} x_{j} s\right),
$$


we use the result in (11) for $V=Z$ here with semi-analytical methods and structure functions from Ref. [24]. See also the work of Refs. [18-23]. A Monte Carlo realization will appear elsewhere [25], wherein we will ultimately use HERWIG [26], PYTHIA [27] and /or the new shower algorithm in Ref. [29] in lieu of the $\left\{F_{i}\right\}$ and thereby, in principle, improve on the shower/exact result combination in Ref. [28]. Due to its lack of the appropriate color coherence [30], we do not consider ISAJET [31] here.

We compute, with and without QED, the ratio $r_{\exp }=\sigma_{\exp } / \sigma_{B o r n}$ to get the results (We stress that we do not use the narrow resonance approximation here.)

$$
r_{\exp }= \begin{cases}1.1901 & , \mathrm{QCED} \equiv \mathrm{QCD}+\mathrm{QED}, \mathrm{LHC} \\ 1.1872 & , \mathrm{QCD}, \mathrm{LHC} \\ 1.1911 & , \mathrm{QCED} \equiv \mathrm{QCD}+\mathrm{QED}, \text { Tevatron } \\ 1.1879 & , \mathrm{QCD}, \text { Tevatron } .\end{cases}
$$

QED is at the level of $.3 \%$ at both $\mathrm{LHC}$ and FNAL ${ }^{\mathrm{a}}$. We agree with the results in Refs. [18-22]. The QED effect is similar in size to structure function results in Refs. [3-7].

\section{Acknowledgments}

Two of us ( S.J. and B.F.L.W.) thank Profs. S. Bethke and L. Stodolsky for the support and kind hospitality of the MPI, Munich, while a part of this work was completed. This work was supported partly by US DoE contract DE-FG05-91ER40627 and by NATO grants PST.CLG.97751,980342.

\section{References}

1. J. G. da Costa, in Proc. ICHEP04, in press; D. Denisov, , ibid..

2. See for example T. Gehrmann, in Proc. LP2003, ed. C.Sazama ( FNAL, 2004 ), in press; J. Stirling, in Proc. ICHEP04, in press, and references therein.

3. S. Haywood, P.R. Hobson, W. Hollik and Z. Kunszt, in Proc. 1999 CERN Workshop on Standard Model Physics ( and more) at the LHC, CERN-2000-004, eds. G. Altarelli and M.L. Mangano,( CERN, Geneva, 2000 ) p. 122.

4. H. Spiesberger,Phys. Rev. D52, 4936 (1995).

5. W.J. Stirling,"Electroweak Effects in Parton Distribution Functions", talk presented at ESF Exploratory Workshop, Electroweak Radiative Corrections to Hadronic Observables at TeV Energies, Durham, Sept., 2003.

6. M. Roth and S. Weinzierl,Phys. Lett. B590, 190 (2004).

7. J. Stirling, in Proc. ICHEP04, in press.

8. D. R. Yennie, S. C. Frautschi, and H. Suura,Ann. Phys. 13, 379 (1961); see also K. T. Mahanthappa, Phys. Rev. 126, 329 (1962), for a related analysis.

9. See also S. Jadach et al., Comput. Phys. Commun. 102, 229 (1997); S. Jadach, M. Skrzypek and B.F.L. Ward,Phys.Rev. D55, 1206 (1997); S. Jadach, B.F.L. Ward and Z. Was,Phys. Rev. D63, 113009 (2001); S. Jadach, B.F.L. Ward and Z. Was, Comp. Phys. Commun. 130, 260 (2000); S. Jadach et al., ibid.140, 432, 475 (2001).

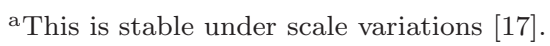


10. M. Dittmar, F. Pauss and D. Zurcher,Phys. Rev. D56, 7284 (1997); M. Rijssenbeek, in Proc. HCP2002, ed. M. Erdmann,( Karlsruhe, 2002 )p. 424; M. Dittmar, ibid.,p.431.

11. S. Klimenko, in Proc. HCP2002, ed. M. Erdmann,( Karlsruhe, 2002 ) p.413.

12. B.F.L. Ward,Phys. Rev. D36, 939 (1987).

13. B.F.L. Ward and S. Jadach, Acta Phys.Polon. B33, 1543 (2002); in Proc. ICHEP2002, ed. S. Bentvelsen et al.,( North Holland, Amsterdam, 2003 ) p. 275.

14. B.F.L. Ward and S. Jadach,Mod. Phys. Lett. A 14, 491 (1999).

15. G. Sterman,Nucl. Phys.B 281, 310 (1987).

16. S. Catani and L. Trentadue, Nucl. Phys.B 327, 323 (1989); ibid. 353, 183 (1991).

17. C. Glosser et al.,Mod. Phys. Lett. A 19, 2119 (2004).

18. U. Baur et al., Phys. Rev. D 57, 199 (1998); ibid.59, 013002 (1998); ibid.65, 033007 (2002), and references therein.

19. S. Dittmaier and M. Kramer, Phys. Rev. D65, 073007 (2002), and references therein.

20. Z. A. Zykunov,Eur. Phys. J.C3, 9 (2001), and references therein.

21. R. Hamberg, W. L. van Neerven and T Matsuura, Nucl. Phys.B359, 343 (1991).

22. W.L. van Neerven and E.B. Zijlstra, Nucl. Phys. B382 (1992) 11; ibid.680, 513 (2004).

23. C. Anastasiou et al.,Phys. Rev. D69,094008 (2004).

24. A.D. Martin et al., Phys. Rev. D51, 4756 (1995).

25. S. Jadach et al., to appear.

26. G. Corcella et al., hep-ph/0210213 and refrences therein.

27. T. Sjostrand et al., hep-ph/0308153

28. S. Frixione and B. Webber, hep-ph/0402116 and references therein.

29. S. Jadach and M. Skrzypek, Acta Phys. Pol. B35, 735 (2004).

30. M. Michelangelo, private communication, 2004.

31. F. Paige et al., hep-ph/0312045 and references therein. 